\title{
The use of castor oil and ricinoleic acid in lead chalcogenide nanocrystal synthesis
}

\author{
Joseph W. M. Kyobe ${ }^{1,2,3} \cdot$ Egid B. Mubofu ${ }^{1}$ - Yahya M. M. Makame ${ }^{1}$ • \\ Sixberth Mlowe $^{2} \cdot$ Neerish Revaprasadu $^{2}$ (D)
}

Received: 2 February 2016/Accepted: 16 August 2016/Published online: 23 August 2016

(c) The Author(s) 2016. This article is published with open access at Springerlink.com

\begin{abstract}
A green solution-based thermolysis method for the synthesis of lead chalcogenide $(\mathrm{PbE}, \mathrm{E}=\mathrm{S}, \mathrm{Se}, \mathrm{Te})$ nanocrystals in castor oil (CSTO) and its isolate ricinoleic acid (RA) is described. The blue shift observed from the optical spectra of CSTO and RA-capped PbE nanocrystals (NCs) confirmed the evidence of quantum confinement. The dimensions of PbE NCs obtained from NIR absorption spectra, transmission electron microscopy (TEM), and $\mathrm{X}$-ray diffraction (XRD) studies were in good agreement. The particle sizes estimated were in the range of 20, 25, and $130 \mathrm{~nm}$ for castor oil-capped $\mathrm{PbS}, \mathrm{PbSe}$, and $\mathrm{PbTe}$, respectively. Well-defined close to cubic-shaped particles were observed in the scanning electron microscopy (SEM) images of $\mathrm{PbSe}$ and $\mathrm{PbTe}$ nanocrystals. The high-resolution TEM and selective area electron diffraction (SAED) micrographs of the as-synthesized crystalline PbE NCs showed distinct lattice fringes with d-spacing distances corroborating with the standard values reported in literature.
\end{abstract}

Keywords Castor oil · Ricinoleic acid · Lead chalcogenide nanocrystals · Optical properties · Green synthesis

Neerish Revaprasadu

RevaprasaduN@unizulu.ac.za

1 Department of Chemistry, University of Dar es Salaam, P.O. Box 35061, Dar es Salaam, Tanzania

2 Department of Chemistry, University of Zululand, Private Bag X1001, Kwa-Dlangezwa 3886, South Africa

3 Department of Chemistry, St. John's University of Tanzania, P.O. Box 47, Dodoma, Tanzania

\section{Introduction}

In recent years, there has been an increase in the use of greener chemical protocols for the synthesis of nanomaterials. The key principles in synthetic routes to nanomaterials include the minimization of inherent toxicity of nanomaterials to health, reduction of ecotoxicity, and minimization of the energy of operational costs during the synthesis processes [1]. Coordinating solvents or stabilizing agents such as alkylamines, e.g., hexadecylamine or phosphines such as trioctylphosphine oxide (TOPO), are examples of inherently toxic materials used in synthetic procedures [2]. Researchers have now focused their attention on developing synthetic protocols to nanomaterials without making use of auxiliary harsh solvents, reducing agents, or capping agents [3-5].

Lead chalcogenides are direct bandgap IV-VI semiconductors, which exhibit unusual physical properties such as an anomalous order of bandgaps, high carrier mobility, high dielectric constant, optical photosensitivity near infrared, strong quantum size effect, multiple exciton generation, and positive temperature coefficients [6-9]. Nanostructured lead chalcogenides are excellent materials for increasing thermoelectric efficiency [10-13], photovoltaic devices [6], biological imaging [14], and infrared detectors [15]. The wide applications of lead chalcogenides $(\mathrm{PbS}, \mathrm{PbSe}$, and $\mathrm{PbTe} \mathrm{NCs}$ ) are because of their ability to produce multiple excitons upon absorbing a single proton of appropriate energy, a process known as multiple exciton generation (MEG). For example, it has been anticipated that when $\mathrm{PbE}(\mathrm{E}=\mathrm{S}, \mathrm{Se}, \mathrm{Te})$ nanocrystals are bombarded with photons of energy, their bandgap energies generate up to three excitons and high quantum yield up to $300 \%$ $[16,17]$. This several-fold multiplicity of electron-hole pairs per absorbed photon has significant effect on power 
conversion efficiencies in photovoltaic cells and other technological devices such as light emitting diodes, lasers, and bipolar transistors.

The use of precursors in the form of metal salts and chalcogenide sources or single molecular entities containing both metal and chalcogenides is an effective route to lead chalcogenides [18-27]. This so-called hot injection precursor route involves the fast injection of solution single or dual source precursors dispersed in a coordinating solvent into a pre-heated capping agent. The first report on the synthesis of well-controlled lead selenide nanocrystals was reported by Murray and his co-workers using lead oleate and $n$-trioctylphosphine selenide (TOPSe) as precursors and diphenylether-TOP as medium via the hot injection method [18]. Hines and Scholes reported the first synthesis of colloidal infrared emitting $\mathrm{PbSe}$ with fine-size distribution [19]. The synthesis of $\mathrm{PbS}$ nanostructures using single source complexes has been widely reported [20-24]. PbTe nanostructures in the form of spheres, cubes, rods, and nanowires have been reported by Revaprasadu and co-workers [25-27].

The surface passivation of $\mathrm{PbE}(\mathrm{E}=\mathrm{S}, \mathrm{Se}, \mathrm{Te})$ nanocrystals with different organic molecules (ligands) is carried out to saturate all surface dangling bonds leading to an improvement in their optical and optoelectronic properties. The capping agents also play a significant role in the growth of nanocrystals and determine the size and shape of the nanocrystals. Despite such significant and excellent electronic passivation behavior, most of the capping and solvent molecules used (e.g., TOPO, alkylamines) are harsh, nasty, inherently toxic, air sensitive, and expensive [20]. As a result, coverage of surfaces of nanocrystals with such materials can be hazardous when used in different applications. In this paper, $\mathrm{PbS}, \mathrm{PbSe}$, and $\mathrm{PbTe}$ nanocrystals were synthesized using a solution-based thermolysis route using castor oil/ricinoleic acid as both dispersing and coordinating solvent. Castor oil and 12-hydroxyl-9-octadecenoic acid (ricinoleic acid) are both naturally occurring bio-based oils that are non-toxic and hence their use as solvents renders the synthetic route greener. There have been reports of the use of other green materials such as anacardic acid [4], olive oil [28], castor oil, and ricinoleic acid [29] for the synthesis of lead chalcogenides. In this study, lead chalcogenide materials were prepared using similar methods.

\section{Experimental}

\section{Materials}

All chemicals used were of high purity (analytical grade). Lead chloride, lead acetate, sulfur powder, selenium powder, methanol, and acetone were purchased from Sigma-Aldrich and all were used in their pure form.
Sodium borohydride and tellurium powder were supplied by Merck and were also used in their analytical-grade forms. Castor seeds were collected from Dodoma and Iringa regions in Tanzania. The castor oil which comprises a high percentage of ricinoleic acid $(\mathrm{C} 18: 1-1 \mathrm{OH})$ was extracted from castor seeds by the Soxhlet (solvent extraction) method. The ricinoleic acid was isolated from castor oil using the method described elsewhere [30].

\section{General procedure for the synthesis of $\mathrm{PbE}$ $(\mathrm{E}=\mathrm{S}$, Se, Te) chalcogenides}

At room temperature, $0.01032 \mathrm{~g}(0.322 \mathrm{mmol})$ of sulfur, $0.0254 \mathrm{~g} \quad(0.322 \mathrm{mmol})$ selenium, or $0.0411 \mathrm{~g}$ $(0.322 \mathrm{mmol})$ tellurium powders were separately added to $0.032 \mathrm{~g}(0.8459 \mathrm{mmol})$ of $\mathrm{NaBH}_{4}$ dissolved in $20 \mathrm{~mL}$ distilled water in three necked bottles. The mixtures were magnetically stirred under purging inert atmosphere for $2 \mathrm{~h} .0 .1221 \mathrm{~g}(0.322 \mathrm{mmol})$ of lead (II) acetate salt dissolved in $20 \mathrm{~mL}$ distilled water was separately added to the reduced solutions of $\mathrm{S}, \mathrm{Se}$, or $\mathrm{Te}$, and the resulting mixture was stirred further for the next $30 \mathrm{~min}$. Excess anhydrous methanol was then added to precipitate $\mathrm{PbE}(\mathrm{E}=\mathrm{S}$, Se, or $\mathrm{Te})$. The precipitated bulk PbE colloids were isolated by centrifugation, dried to remove the solvent, and kept for the next step.

\section{Synthesis of $\mathrm{PbE}(\mathrm{E}=\mathrm{S}$, Se, Te) nanocrystals}

A solution of $\mathrm{PbE}$ (E: $\mathrm{S}, \mathrm{Se}, \mathrm{Te})$ dispersed in a coordinating solvent was rapidly injected into a hot and vigorously magnetically stirred $6.0 \mathrm{~g}$ castor oil or ricinoleic acid (ricinoleic acid was used only for the synthesis of $\mathrm{PbS}$ ) preheated at $280{ }^{\circ} \mathrm{C}$. The reaction mixture under reflux was thermalized for $2 \mathrm{~h}$, and aliquot samples were removed after 30, 60, and $120 \mathrm{~min}$. Excess anhydrous methanol was immediately added to the samples to flocculate the $\mathrm{PbE}$ NCs. The flocculates were separated by centrifugation and completely re-dispersed in acetone to produce an optically clear colloidal solution for characterization. The overall mechanism (profile) for synthesis of $\mathrm{PbE} \mathrm{NCs} \mathrm{is}$ schematically illustrated in Fig. 1.

\section{Characterization}

Sample characterization was performed using a PerkinElmer Lambda 1050 UV-Visible NIR spectrometer. The samples were placed in silica cuvettes ( $1 \mathrm{~cm}$ path length), using acetone as a reference solvent. The structural features of the PbE nanocrystals were analyzed using a JEOL1010 Transmissions Electron Microscope (TEM) and a high-resolution electron microscope (HRTEM) coupled with JEOL 


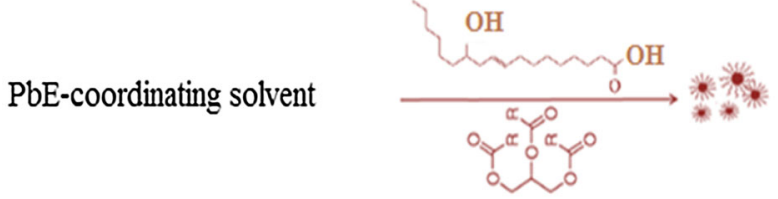

Nanocrystals size selective precipitation in polar solvent

Self assembly (centrifugation) and evaporation of solvent

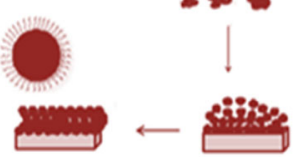

Fig. 1 Reaction profile for the synthesis of castor oil/ricinoleic acidcapped $\mathrm{PbE}(\mathrm{E}=\mathrm{S}$, Se or $\mathrm{Te})$ nanocrystals

2010 TEM operating at an accelerating potential of 100 and $200 \mathrm{kV}$, respectively. Samples for TEM and HRTEM were prepared by deposition of dilute colloidal solutions of $\mathrm{PbE}$ on a carbon-coated disc (grid) and left to dry at room temperature. X-ray diffraction studies to identify the crystalline phase were performed using a Bruker AXS D8 diffractometer equipped with nickel-filtered $\mathrm{CuK} \alpha$ radiation $(\lambda=1.5406 \AA)$ operating at $40 \mathrm{kV}, 40 \mathrm{~mA}$ at room temperature. To ensure a complete removal of excess capping agents, samples for XRD were prepared by repeatedly washing precipitated $\mathrm{PbE}$ nanocrystals with excess acetone and thereafter dried at room temperature to get a dry powder. The dried powdered sample was deposited on a glass substrate (disc) and the $2 \theta$ range was from $5^{\circ}$ to $90^{\circ}$ in a scan rate of 0.2 per second. For detailed surface morphology analysis of PbE nanocrystals, a Zeiss Ultra Plus FEG SEM, equipped with Oxford detector EDX at $20 \mathrm{kV}$, with Aztec software was used. Before being analyzed, the samples were carbon coated using Quorum Coater (Model Q150TE).

\section{Results and discussion}

\section{$\mathrm{PbS}$ nanocrystals}

Castor oil and ricinoleic acid are both liquid at room temperature and their boiling points are 313 and $412{ }^{\circ} \mathrm{C}$, respectively, making them good capping materials to be used in place of air-sensitive, toxic, and inexpensive chemicals or solvents. Castor oil and ricinoleic acid-capped $\mathrm{PbS}$ nanocrystals were synthesized via a solution-based thermolysis using lead acetate salt and elemental sulfur as lead and sulfur precursors. The isolated solid product dispersed in castor oil or ricinoleic acid was rapidly injected into a $6.0 \mathrm{~g}$ castor oil or ricinoleic acid pre-heated at $280{ }^{\circ} \mathrm{C}$. The mixture was thermolyzed for $2 \mathrm{~h}$ under stirring, with aliquots taken out from the reaction mixture after $1 / 2,1$, and $2 \mathrm{~h}$ time intervals. Excess anhydrous methanol was immediately added to the centrifuging tubes to isolate or precipitate the $\mathrm{PbS}$ nanocrystals. The sequence of reactions is shown below:

$$
\begin{aligned}
& 2 \mathrm{~S}(\mathrm{~s})+\mathrm{NaBH}_{4}(\mathrm{~s})+7 \mathrm{H}_{2} \mathrm{O}(\mathrm{l}) \rightarrow 2 \mathrm{NaHS}(\mathrm{aq}) \\
& \quad+\mathrm{Na}_{2} \mathrm{~B}_{4} \mathrm{O}_{7}(\mathrm{aq})+14 \mathrm{H}_{2}(\mathrm{~g}), \mathrm{NaHS}(\mathrm{aq})+\mathrm{PbCl}_{2}(\mathrm{aq}) \\
& \quad \rightarrow \mathrm{PbS}(\mathrm{s})+\mathrm{NaCl}(\mathrm{aq})+\mathrm{HCl}(\mathrm{aq}) .
\end{aligned}
$$

Figure 2 shows the room temperature absorption UVNIR spectra for the $\mathrm{PbS}$ synthesized in castor oil and ricinoleic acid at $280{ }^{\circ} \mathrm{C}$. The absorption spectra show relatively sharp absorption excitonic peaks at approximately 1875, 1895, and $1930 \mathrm{~nm}$ for CSTO-capped PbS NCs (Fig. 2a) and 1900, 2015, and $2045 \mathrm{~nm}$ for RA-capped PbS NCs (Fig. 2b). These excitonic peaks show large quantum confinement inducing blue shift of the absorption edges compared to bulk $\mathrm{PbS}$ (3100 nm). The blueshift decreases with increasing growth period. The size of the $\mathrm{PbS}$ particles was calculated using Eq. 1 [31] on the basis of absorption excitonic peaks (nm) deduced from Fig. 2a, b.

$E_{g}=E_{g}^{b}+\frac{1}{a S^{2}+b S}$,

where $E_{g}$ is the bandgap of $\mathrm{PbS}$ nanocrystals produced, $E_{g}^{b}=0.41 \mathrm{eV}$ is the bulk bandgap [32], and $a=0.0252$, $b=0.283$ are arbitrary constants for $\mathrm{PbS}$, and $\mathrm{S}$ is the size in $\mathrm{nm}$. The calculated mean diameters of the CSTO-capped $\mathrm{PbS}$ NCs were 19.33, 19.54, and $19.84 \mathrm{~nm}$ (Fig. 2a), whereas the RA-capped $\mathrm{PbS}$ had mean diameters of 19.6, 20.6, and $21.0 \mathrm{~nm}$ (Fig. 2b). The difference of $0.8 \mathrm{~nm}$ in
Fig. 2 Optical absorption spectra of nanodispersed $\mathrm{PbS}$ in toluene, synthesized in a castor oil and $\mathbf{b}$ ricinoleic acid at $280{ }^{\circ} \mathrm{C}$
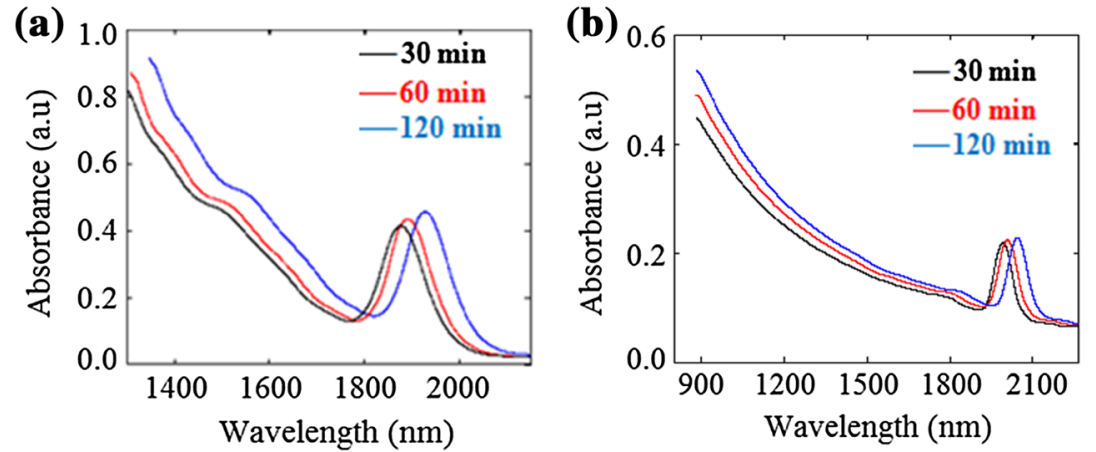


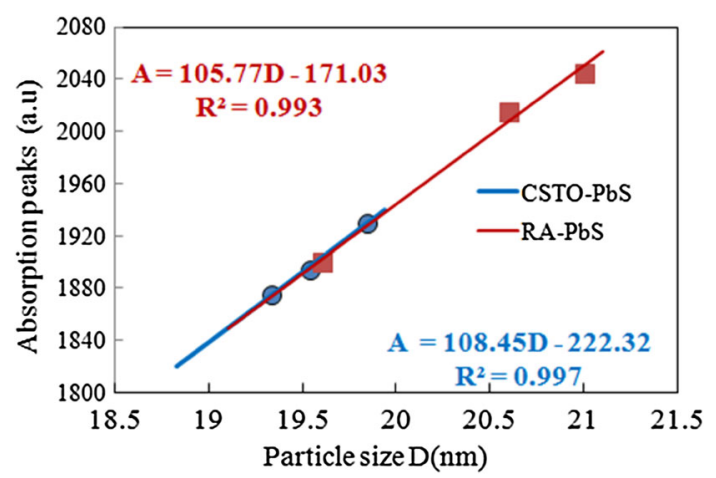

Fig. 3 Plots of absorption peaks against particle size (diameter) for CSTO and RA-capped PbS nanocrystals as prepared at 230, 250 and $280{ }^{\circ} \mathrm{C}$ for $2 \mathrm{~h}$

the average diameter indicates that the growth and redistribution of $\mathrm{PbS} \mathrm{NCs}$ is faster in ricinoleic acid than in castor oil.

Typical features of the absorption peaks versus particle size D (nm) plots are demonstrated in Fig. 3 for castor oil (CSTO) and ricinoleic acid (RA)-capped PbS nanocrystals as synthesized at $280{ }^{\circ} \mathrm{C}$. The notable observation of absorption peaks and the corresponding particle sizes are linearly related. Small values for the absorption peaks of CSTO-capped $\mathrm{PbS}$ nanocrystals indicate that the strength of the covalent attachment of CSTO to surfaces of $\mathrm{PbS}$ is better than RA. This observation was very useful for modeling the treatment of CSTO and RA as eco-friendly capping materials to preferentially bind to the surface of $\mathrm{PbS}$ nanocrystals with different binding energies. The difference in binding energy is, however, small to selectively abandon ricinoleic acid as capping agent as suggested by slopes of the regression lines (Fig. 3). Castor oil (CSTO) and ricinoleic acid (RA) are efficient capping materials capable of performing the duty of stopping uncontrolled growth and agglomeration, particle morphology, particle distribution, and tuning the availability of reagent (nucleation) in the same way as air-sensitive and toxic solvents and materials, which have been frequently used.

The TEM images of castor oil- and ricinoleic acidcapped $\mathrm{PbS}$ nanoparticles show particles with almost cubic morphology (Fig. 4a, b). The estimated mean particle diameters for castor oil- and ricinoleic acid-capped $\mathrm{PbS}$ are $20.08 \pm 1.63$ and $19.70 \pm 1.40 \mathrm{~nm}$, respectively, which is in good agreement with the value obtained from the absorption measurements. The particles have well-defined surfaces which can be observed from high-magnification HRTEM image (Fig. 4b, d). The regular fringes spacing with 0.3457 and $0.3389 \mathrm{~nm}$ for castor oil- and ricinoleic acid-capped $\mathrm{PbS}$ nanoparticles, respectively, correspond to those (200) lattice planes of the face-center cubic phase of $\mathrm{PbS}$.
Fig. 4 TEM and HRTEM images of as-prepared castor oil- $(\mathbf{a}, \mathbf{b})$ and ricinoleic acid(c, d) capped $\mathrm{PbS} \mathrm{NCs}$ at $280{ }^{\circ} \mathrm{C}$ for $2 \mathrm{~h}$
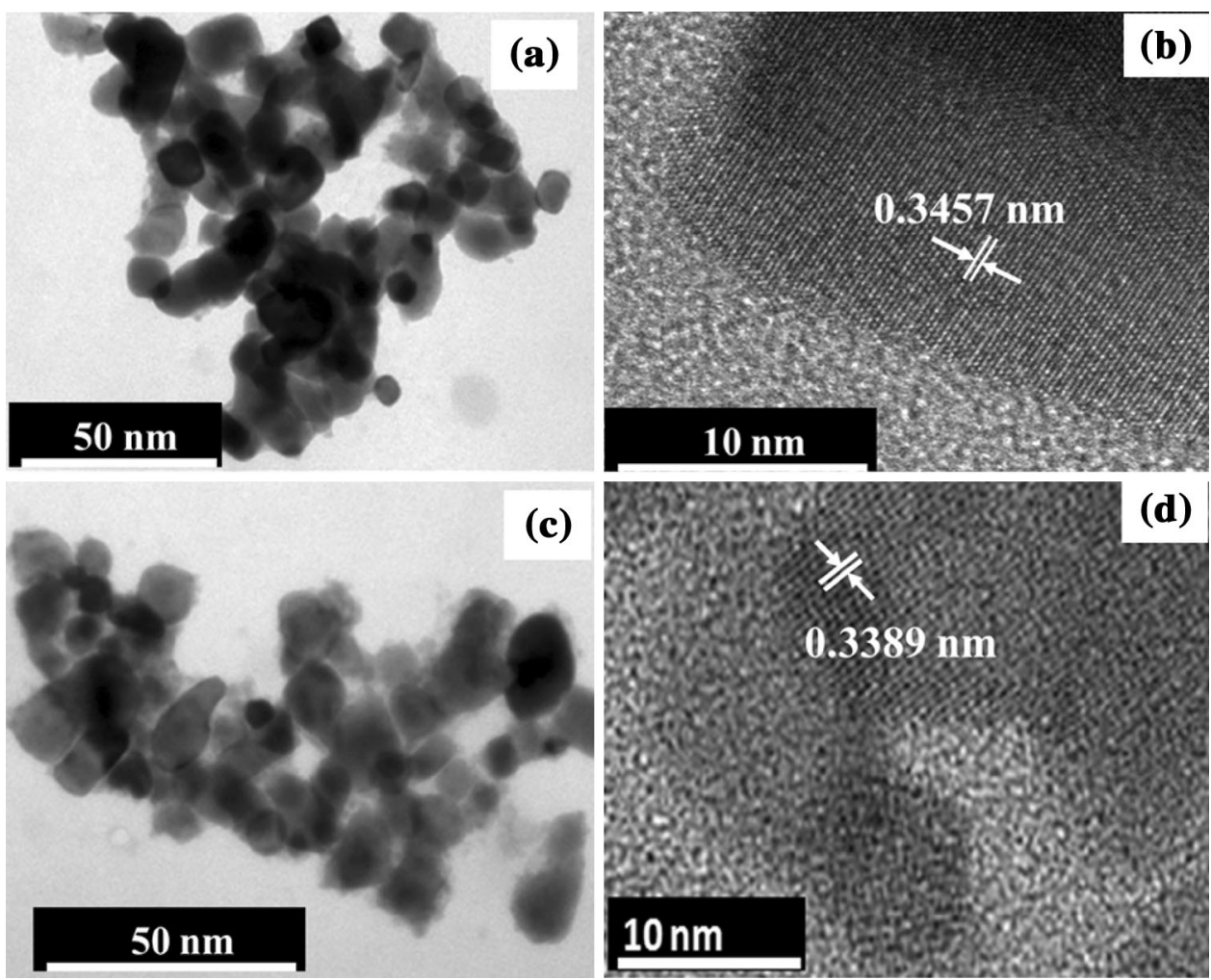


\section{PbSe nanocrystals}

We prepared PbSe nanocrystals capped by castor oil by injecting a bulk PbSe dispersed in CSTO into a magnetically agitated hot CSTO at 280 C, followed by thermolysis for $2 \mathrm{~h}$. Upon injection, small $\mathrm{PbSe}$ seeds or clusters immediately nucleated with the growth process monitored similar to that of $\mathrm{PbS}$ nanoparticles. Figure 5a shows the absorption spectra of CSTO-capped PbSe NCs with the first excitonic peaks appearing at 1820, 1850 and $1910 \mathrm{~nm}$. The peaks were well defined, reflecting the narrow size distribution of the particles. As a large number of nucleation centers are initially formed, the castor oil prevents or limits particle growth via Ostwald ripening. The mean particle size $S$ (nm) of as-synthesized PbSe NCs was calculated from Eq. 2 [33].

$E_{g}^{n}=E_{g}^{b}+\left(0.016 S^{2}+0.209 S+0.45\right)^{-1}$,

where $S$ is the mean particle size $(\mathrm{nm}), E_{g}^{n}$ is the energy of the first exciton absorption peak, which varies with $1 / \mathrm{S}$, and $E_{g}^{b}=0.28 \mathrm{eV}$ is the bulk bandgap energy [34].

The equation was established by relating the TEM image diameters to the bandgap energy of the first excitonic transition recorded with absorbance spectroscopy. Using Eq. 2 and absorption peaks from Fig. 5a, we calculated 19.6, 19.74 and $20.1 \mathrm{~nm}$ as mean particle sizes of as-synthesized CTSO-PbSe. Figure 5b shows PbSe NCs with a cube-like morphology with an average size of $25.52 \pm 1.34 \mathrm{~nm}$. The corresponding high-resolution TEM image of the CSTO-capped PbSe shows that the NCs formed are crystalline, with distinct lattice fringes and a lattice d-spacing of $0.3467 \mathrm{~nm}$ (Fig. 5c). The SEM image of the CSTO-capped PbSe also shows particles in the form of cubes in the range of 180-200 nm (Fig. 5d).

\section{PbTe nanocrystals}

We also synthesized PbTe nanoparticles in castor oil using similar reaction conditions as that of $\mathrm{PbSe}$. Figure $6 \mathrm{a}$ shows the absorption spectra of PbTe NCs displaying the first excitonic absorption peak at approximately 2085, 2115 , and $2180 \mathrm{~nm}$ for the 30, 60, and $120 \mathrm{~min}$ samples. From the TEM images (Fig. 6b), we noticed cubic-tospherical morphology of the as-synthesized castor oilcapped PbTe NCs with an average particle size of $129.53 \pm 10.27 \mathrm{~nm}$. The SEM image shows well-defined PbTe NCs assembled as perfect cubes (Fig. 6c). The selective area electron diffraction (SAED) pattern, as in Fig. 6d, shows that all detectable dot rings perfectly reveal the formation of highly crystalline PbTe nanocrystals.
Fig. 5 a UV-NIR absorption spectra, b TEM, c HRTEM and d SEM image of as-synthesized castor oil-PbSe nanocrystals grown at $280{ }^{\circ} \mathrm{C}$ for $2 \mathrm{~h}$
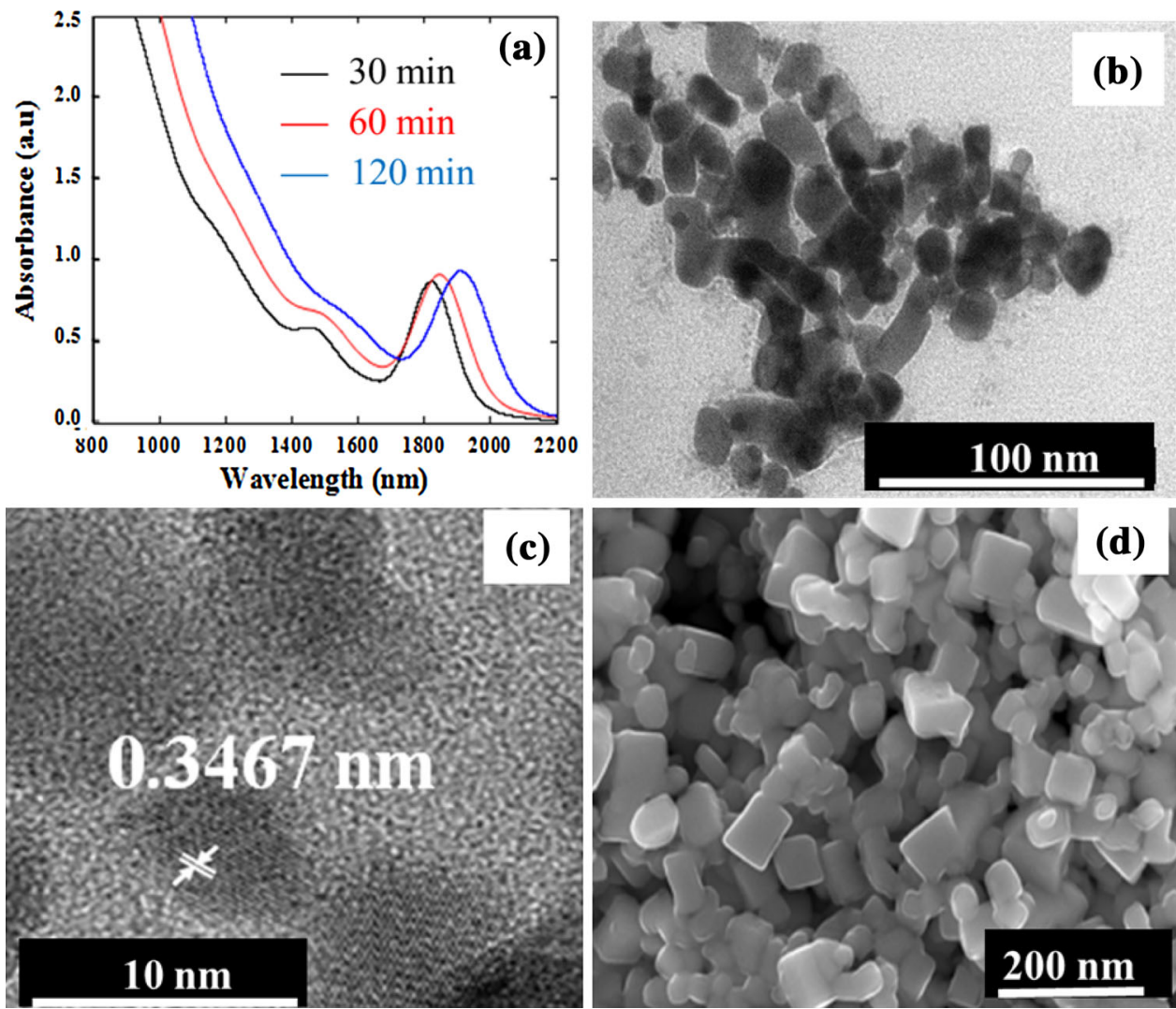
Fig. 6 a UV-NIR absorption spectra, b TEM, c SEM and d SAED images of assynthesized castor oil-PbTe nanocrystals grown at $280{ }^{\circ} \mathrm{C}$ for $2 \mathrm{~h}$
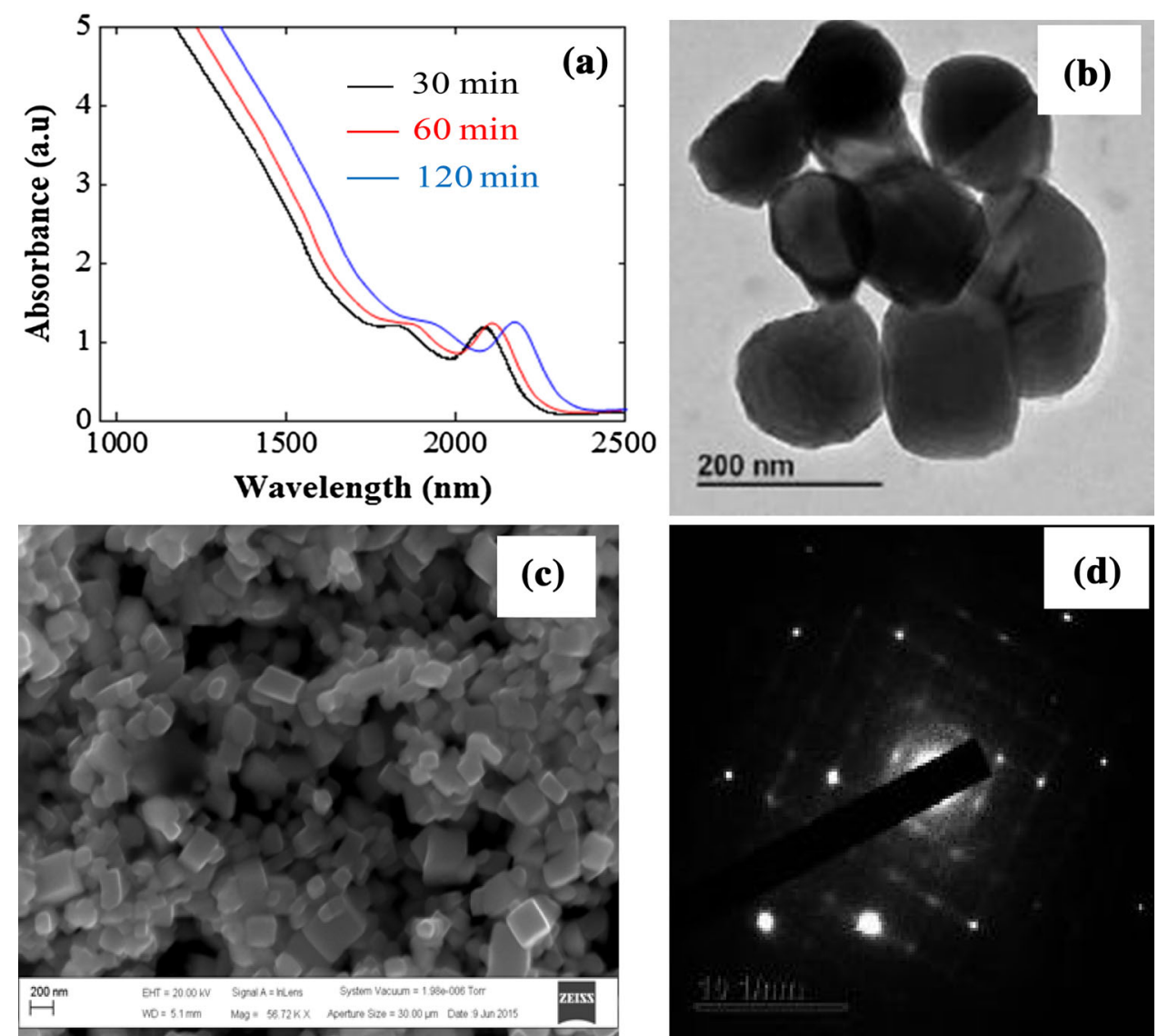

\section{Structural properties}

The XRD pattern of PbS NCs prepared in castor oil and ricinoleic acid is shown in Fig. 7. There are distinct peaks indexed to the (111), (200), (220), (222), (420), and (422) planes of the cubic rock salt structure (card number: 78-1898). The high intensity of the (200) peak shows that

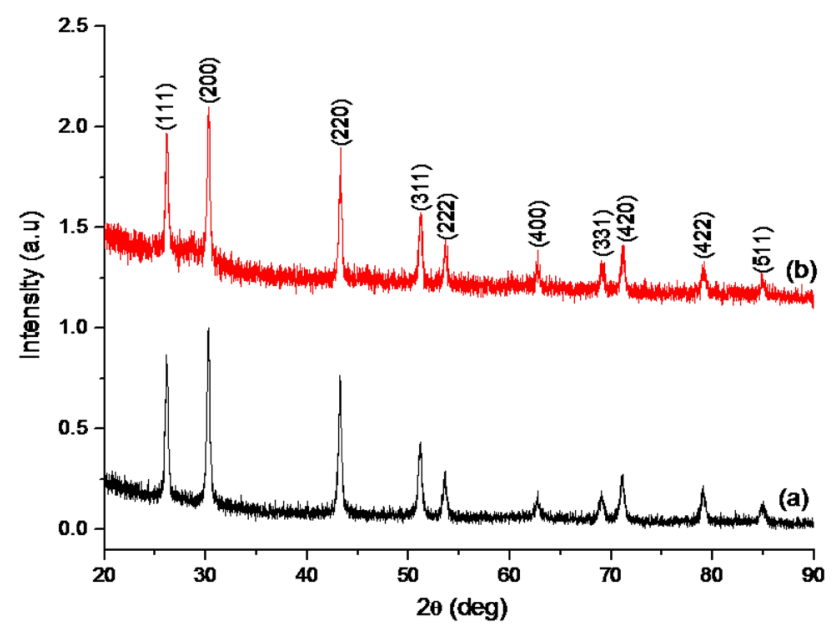

Fig. 7 XRD patterns of $\mathrm{PbS}$ nanocrystals prepared in a ricinoleic acid; $\mathbf{b}$ castor oil at $280^{\circ} \mathrm{C}$ for $2 \mathrm{~h}$ growth time
$\mathrm{PbS}$ NCs have a large number of (200) planes, making it the dominant reflection in the diffraction feature. The sizes of $\mathrm{PbS}$ NCs calculated using Scherrer equation [35] along the (200) peak is 22.62 for CSTO-capped $\mathrm{PbS}$ and $20.50 \mathrm{~nm}$ for ricinoleic acid-capped $\mathrm{PbS}$ which is in good agreement with the values determined from TEM measurements (Fig. 4).

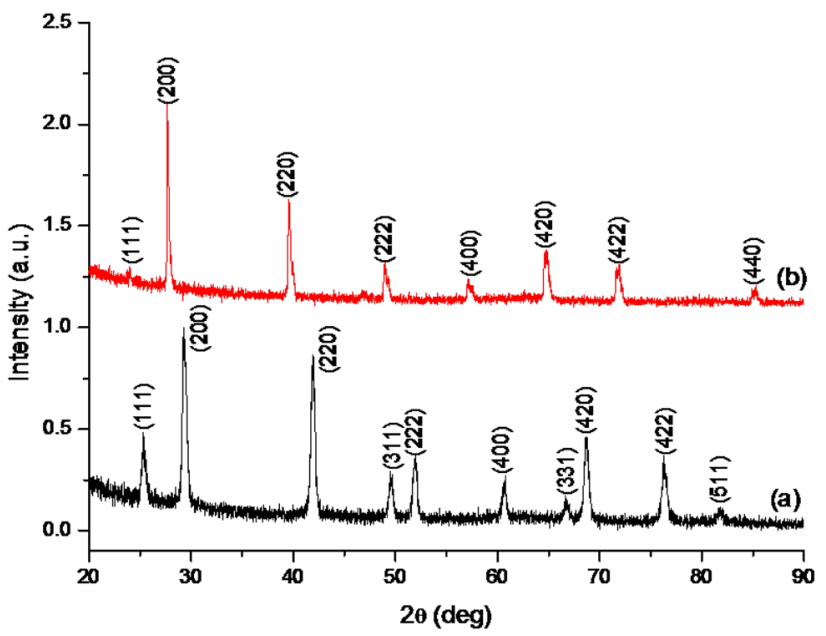

Fig. 8 XRD pattern of castor oil-capped a PbSe and b PbTe NCs synthesized at $280{ }^{\circ} \mathrm{C}$ 
Figure 8 shows room temperature XRD patterns of (a) PbSe and (b) PbTe NCs as synthesized in castor oil at 280 C. The (111), (200), (220), (222), (420), and (422) reflection planes observed are indexed to the cubic phase of $\mathrm{PbSe}$ and PbTe. The narrow features of the (200) plane further reveal the large size of the PbTe NCs, as confirmed by the TEM measurements.

\section{Conclusion}

We have presented a simple, cheap and greener route to synthesize lead chalcogenide (PbE) nanocrystals (NCs) interfaced with castor oil and ricinoleic acid. Castor oil and ricinoleic acid, liquids at room temperature, are non-air sensitive, non-toxic, relatively cheap and have a high boiling point. $\mathrm{PbS}, \mathrm{PbSe}$ and $\mathrm{PbTe}$ nanocrystals prepared showed the formation of spherical-to-cubic shapes with varying sizes, ranging from 20 to $130 \mathrm{~nm}$. Powder X-ray diffraction patterns revealed the formation of pure crystalline cubic phase for all samples. The optical properties of the as-synthesized lead chalcogenide nanocrystals exhibited strong quantum confinement properties. The optical behavior of the nanocrystals further revealed that the absorption properties were temperature dependent. Castor oil and ricinoleic acid have been identified as naturally occurring biobased organic compounds, which can be effectively used in the synthesis of lead chalcogenide nanomaterials.

Acknowledgments The authors are grateful to the National Research Foundation (NRF) through the South African Research Chair initiative (SARChI) and the NRF-COSTECH Project for financial support.

Open Access This article is distributed under the terms of the Creative Commons Attribution 4.0 International License (http://creative commons.org/licenses/by/4.0/), which permits unrestricted use, distribution, and reproduction in any medium, provided you give appropriate credit to the original author(s) and the source, provide a link to the Creative Commons license, and indicate if changes were made.

\section{References}

1. Anastaz, P.T., Warner, J.C.: Green chemistry: theory and practice, p. 30. Oxford University Press, Oxford (1998)

2. Green, M.: The nature of quantum dots capping ligands. J. Mater. Chem. 20, 5797-5808 (2010)

3. Amirjan, A., Bagheri, M., Heydari, M., Hesaraki, S.: Label-free surface Plasmon resonance detection of hydrogen peroxide; a bioinspired approach. Sensor Actuators B. 227, 373-382 (2016)

4. Mlowe, S., Nejo, A.A., Pullabhotla Rajasekhar, V.S.R., Mubofu, E.B., Ngassapa, F.N., O'Brien, P., Revaprasadu, N.: Lead chalcogenides stabilized by anacardic acid. Mater Sci Semicond Process 16, 263-268 (2013)

5. Akhtar, J., Malik, M.A., O'Brien, P., Wijayantha, K.G.U., Dharmadasa, R., Hardman, S.J.O., Graham, D.M., Spencer, B.F., Stubbs, S.K., Flavell, W.R., Binks, D.J., Sirotti, F., Kazzi, M.E.,
Silly, M.: A greener route to photoelectrochemically active $\mathrm{PbS}$ nanoparticles. J. Mater. Chem. 20, 2336-2344 (2010)

6. Fu, H., Tsang, S.-W.: Infrared colloidal lead chalcogenide nanocrystals: synthesis, properties, and photovoltaic applications. Nanoscale. 4, 2187-2201 (2012)

7. Alvi, M.A., Khan, Z.H.: Synthesis and characterization of nanoparticle thin films of $\mathrm{a}-(\mathrm{PbSe}) 100-\mathrm{xCd}_{\mathrm{x}}$ lead chalcogenides. Nanoscale Res. Lett. 8, 148 (2013)

8. Murphy, J.E., Beard, M.C., Norma, A.G., Ahrenkiel, S.P., Johnson, J.C., Yu, P., Micic, O.I., Ellington, R.J., Nozik, A.J.: PbTe colloidal nanocrystals: synthesis, characterization, and multiple exciton generation. J. Am. Chem. Soc. 128, 3241-3247 (2006)

9. Kungumadevi, L., Amoorthy, S.R.: Synthesis of PbTe nanocubes, worm-like structures and nanoparticles by simple thermal evaporation method. Bull. Mat. Sci. 36(15), 771-778 (2013)

10. Wang, H., Gibbs, Z.M., Takagiwa, Y., Snyder, G.J.: Tuning bands of PbSe for better thermoelectric efficiency. Energy Environ Sci 7, 804-811 (2014)

11. Roh, J.W., Jang, S.Y., Kang, J., Lee, S., Noh, J.S., Kim, W., Park, J., Lee, W.: Size-dependent thermal conductivity of individual single-crystalline PbTe nanowires. Appl. Phys. Lett. 96, 103101 (2010)

12. Hazan, E., Ben-Yehuda, O., Madar, N., Gelbstein, Y.: Functional graded germanium-lead chalcogenide-based thermoelectric module for renewable energy applications. Energy Mater, Adv (2015). doi:10.1002/aenm.201500272

13. Yamini, S.A., Ikeda, T., Pei, Y.A.L., Doua, S.X., Snyder, G.J.: Rational design of p-type thermoelectric PbTe: temperature dependent sodium solubility. J. Mater. Chem. A 1(31), 8725-8730 (2013)

14. Bruchez, M., Moronne, M., Gin, P., Wiess, S., Alivisatos, A.P.: Semiconductor nanocrystals as fluorescent biological labels. Science 281, 2013-2016 (1998)

15. Wang, Q., Chen, G., Yin, H.: New insights into the growth mechanism of hierarchical architectures of PbTe synthesized through a triethanolamine-assisted solvothermal method and their shape-dependent electrical transport properties. J. Mater. Chem. A1, 15355-15369 (2013)

16. Schaller, R.D., Klimov, V.I.: High efficiency carrier multiplication in PbSe nanocrystals. Implication for solar energy conversion. Phys. Rev. Lett. 92, 186601-186604 (2004)

17. Ellington, R.J., Beard, M.C., Johnson, J.C., Yu, P., Micic, O.J., Nozik, A.J., Shabaev, A., Efros, A.L.: High efficient multiple exciton generation in colloidal $\mathrm{PbSe}$ and $\mathrm{PbSe}$ quantum dots. Nano Lett. 5, 865-871 (2005)

18. Murray, C.B., Sun, S.H., Gaschler, W., Doyle, H., Betley, T.A., Kagan, C.R.: Colloidal synthesis of nanocrystals and nanocrystal superlattices. IBM J. Res. Dev. 45, 47-56 (2001)

19. Hines, M.A., Scholes, G.D.: Colloidal PbS nanocrystals with size-tunable near-infrared emission: observation of post synthesis self-narrowing of the particle size distribution. Adv. Mater. 15, 1844-1849 (2003)

20. Sapra, S., Rogath, A.L., Feldmann, J.: Phosphine-free synthesis of monodisperse CdSe nanocrystals in olive oil. J. Mater. Chem. 16, 3391-3395 (2006)

21. Zhang, Y.C., Qiao, T., Hu, X.Y., Wang, G.Y., Wu, X.: Shapecontrolled synthesis of $\mathrm{PbS}$ microcrystallites by mild solvothermal decomposition of a single-source molecular precursor. J. Cryst. Growth 277, 518-523 (2005)

22. Trindade, T., O'Brien, P., Zhang, X.-M., Motevalli, M.: Synthesis of $\mathrm{PbS}$ nanocrystallites using a novel single molecule precursors approach: X-ray single-crystal structure of $\mathrm{Pb}\left(\mathrm{S}_{2} \mathrm{CNEtPri}\right)_{2}$. J. Mater. Chem. 7(6), 1011-1016 (1997) 
23. Salavati-Niasaria, M., Sobhani, A., Davara, F.: Synthesis of starshaped $\mathrm{PbS}$ nanocrystals using single-source precursor. J. Alloys Compd. 507, 77-83 (2010)

24. Nyamen, L.D., Rajasekhar Pullabhotla, V.S.R., Nejo, A.A., Ndifon, P.T., Warner, J.H., Revaprasadu, N.: Synthesis of anisotropic $\mathrm{PbS}$ nanoparticles using heterocyclic dithiocarbamate complexes. Dalton Trans 41, 8297-8302 (2012)

25. Revaprasadu, N., Rajasekhar Pullabhotla, V.S.R.: Shape evolution of $\mathrm{PbTe}$ nanostructures using mixed lead sources. Mater. Lett. 97, 108-112 (2013)

26. Ramasamy, K., Nejo, A.O., Ziqubu, N., Rajasekhar Pullabhotla, V.S.R., Nejo, A.A., Revaprasadu, N., O'Brien, P.: A new route to lead chalcogenide nanocrystals. Euro J. Inorg. Chem. 33, 5196-5201 (2011)

27. Ziqubu, N., Ramasamy, K., Rajasekhar Pullabhotla, V.S.R., Revaprasadu, N., O'Brien, P.: Simple route to dots and rods of PbTe nanocrystals. Chem. Mater. 22(13), 3817-3819 (2010)

28. Nyamen, L.D., Revaprasadu, N., Ndifon, P.T.: Low temperature synthesis of $\mathrm{PbS}$ and $\mathrm{CdS}$ nanoparticles in olive oil. Mater Sci. Semicond. Process. 27, 191-196 (2014)

29. Kyobe, J.W., Mubofu, E.B., Makame, Y.M.M., Mlowe, S., Revaprasadu, N.: CdSe quantum dots capped with naturally occurring biobased oils. New J. Chem. 39, 7251-7259 (2015)
30. Vaisman, B., Shikanov, A., Domb, A.J.: The isolation of ricinoleic acid from castor oil by salt-solubility-based fractionation for the biopharmaceutical applications. J. Am. Oil Chem. Soc. 85, 169-184 (2008)

31. Moreels, I., Lambert, K., Smeets, D.De, Muyuk, D., Vanhaecke, F., Vantomme, A., Delerne, C., Allan, G., Hens, Z.: Size-dependent optical properties of PbS quantum dots. ACS Nano 3, 3023-3030 (2009)

32. Grahn, H.T.: Introduction to semiconductor physics. World Scientific, Singapore (1999)

33. Moreels, I., Lambert, K.De, Muyuk, D., Vanhaecke, F., Poelman, D., Martins, J.C., Allan, G., Hens, Z.: Composition and sizedependent extinction coefficient of colloidal $\mathrm{PbSe}$ quantum dots. Chem. Mater. 19, 6101-6106 (2007)

34. Ekuma, C.E., Singh, D.J., Moreno, J., Jarrell, M.: Optical properties of PbTe and PbSe. Phys. Rev. B 85(8), 085205 (2012)

35. Patterson, A.L.: The Scherrer formula for X-Ray particle size determination. Phys. Rev. 56, 978-982 (1939) 